\title{
Prenatal diagnosis and molecular cytogenetic characterization of hereditary complex chromosomal rearrangements in a Chinese family
}

\author{
Dongling Tang ${ }^{1 *}$, Bei Wang ${ }^{2 *}$, Mingxia Xie ${ }^{3 *}$, Zeyu Li ${ }^{4}$ \\ ${ }^{1}$ Department of Clinical Laboratory, Renmin Hospital of Wuhan University, Wuhan, Hubei, China \\ ${ }^{2}$ Department of Gastroenterology, Wuhan Hankou Hospital, Wuhan, Hubei, China \\ ${ }^{3}$ Department of Obstetrics and Gynecology; The 457 Hospital of PLA, Wuhan, Hubei, China \\ ${ }^{4}$ Department of Clinical Laboratory, The First People's Hospital of Yichang, Yichang, Hubei, China \\ *These authors contributed equally to this work
}

\begin{abstract}
Objectives: To report a family with an extremely rare and previously undescribed complex chromosomal rearrangement (CCR). To explore the molecular cytogenetic mechanism of 'octaradial chromosome'.

Material and methods: G-banding karyotype analysis was performed on all the members of the family. Chromosomal microarray analysis(CMA) was performed on the five members of the family.

Results: This case presented with a karyotypically balanced CCR $(46, X X, t(2 ; 4 ; 11 ; 5)(p 21 ; q 34 ; q 21 ; p 15))$. The familial CCR was stably transmitted across three generations.

Conclusions: We report an extremely rare and previously undescribed complex chromosomal arrangement that is transmitted across three generations. The clinical outcome of this CCR is complex. Careful characterization of all the breakpoint regions is required for prenatal diagnosis and genetic counseling.

Key words: Chromosome karyotype; chromosomal microarray analysis (CMA); complex chromosomal rearrangements; octaradial chromosome; prenatal diagnosis
\end{abstract}

Ginekologia Polska 2022; 93, 7: 570-573

\section{INTRODUCTION}

Complex chromosomal rearrangements (CCRs) are rearrangements involving more than two chromosomes or having more than two breakpoints [1]. The occurrence of constitutional CCRs is rare with approximately 250 cases reported so far $[2,3]$. The CCRs detection rate increases with the application of molecular cytogenetic technology. Karyotypically detected complex rearrangements are further analyzed using methods such as chromosomal microarray analysis (CMA), fluorescence in situ hybridization (FISH) and whole-genome sequencing (WGS) [4]. Here we report an extremely rare and previously undescribed CCR that stably is transmitted across three generations.

\section{Objectives}

To report a family with an extremely rare and previously undescribed complex chromosomal rearrangement
(CCR). To explore the molecular cytogenetic mechanism of 'octaradial chromosome'.

\section{MATERIAL AND METHODS}

In 2011, a 24-year-old primigravid woman underwent amniocentesis at 20 weeks of gestation because her adjusted Down syndrome risk was 1/50.

G-banding karyotype analysis was performed on all the members of the family.

CMA has been introduced in clinical diagnosis to rapidly detect genome-wide gains and losses with higher resolution. CMA was performed on the five members of the family.

\section{RESULTS}

Conventional karyotyping using cultured amniocytes revealed a karyotype of $46, \mathrm{XY}, \mathrm{t}(2 ; 4 ; 11 ; 5)(\mathrm{p} 21 ; \mathrm{q} 34 ; \mathrm{q} 21 ; \mathrm{p} 15)$ (Fig. 1). Conventional karyotyping using peripheral blood

Corresponding author:

Zeyu Li

Department of Clinical Laboratory, The First People's Hospital of Yichang, Yichang, Hubei, China e-mail: yuchunjiaofy@163.com 

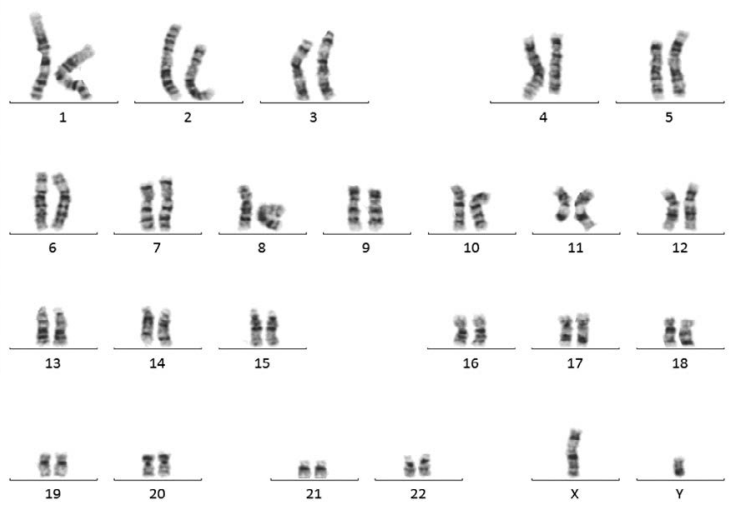

Figure 1. Karyotype of III-1, 46,XY,t(2;4;11;5)(p21;q34;q21;p15)mat
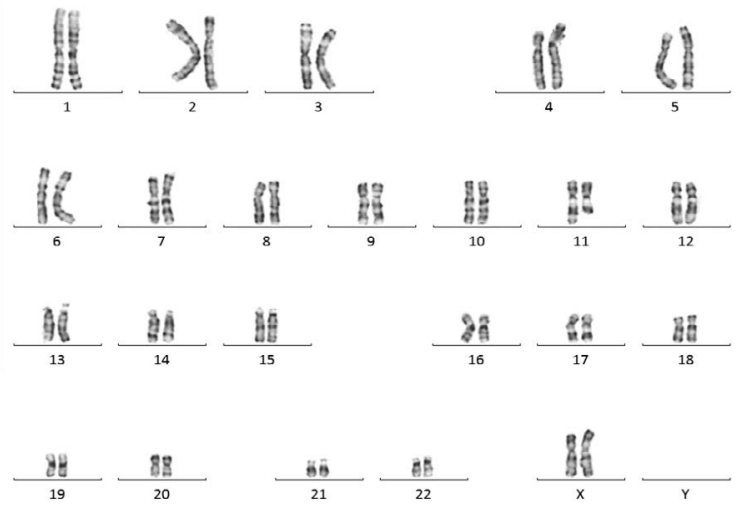

Figure 2. Karyotype of II-2, 46,XX,t(2;4;11;5)(p21;q34;q21;p15)mat

revealed the same chromosome rearrangement in the mother and the maternal grandmother (Fig. 2,3) and a normal karyotype in the father and the maternal grandfather. The pedigree of the family showed that this chromosome rearrangement was stably transmitted across three generations (Fig. 4).

CMA analysis on uncultured amniocytes detected no pathogenic variants. Parental CMA didn't detect any pathogenic variants. Ultrasound examination showed no dysmorphism and intrauterine growth restriction (IUGR) [5] in the fetus. Because both the mother and the grandmother carry the same translocation and have a normal phenotype, we conclude this is a balanced translocation. After genetic counseling, the couple decided to continue the pregnancy. At 38 weeks of gestation, a $3100 \mathrm{~g}$ physically normal male baby was delivered naturally. The infant was phenotypically normal at birth. He had normal growth and psychomotor development by age 9 .

In 2020, this woman was pregnant again and underwent amniocentesis at 20 weeks of gestation. Conventional karyo-

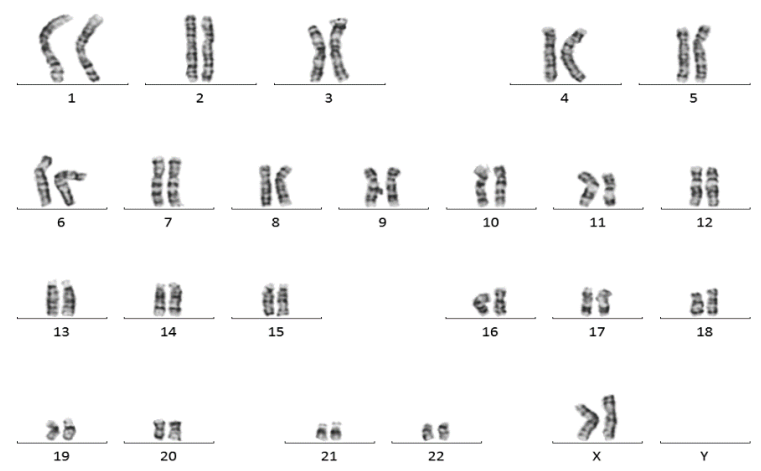

Figure 3. Karyotype of $1-2,46, X X, t(2 ; 4 ; 11 ; 5)(p 21 ; q 34 ; q 21 ; p 15)$
I
II
III

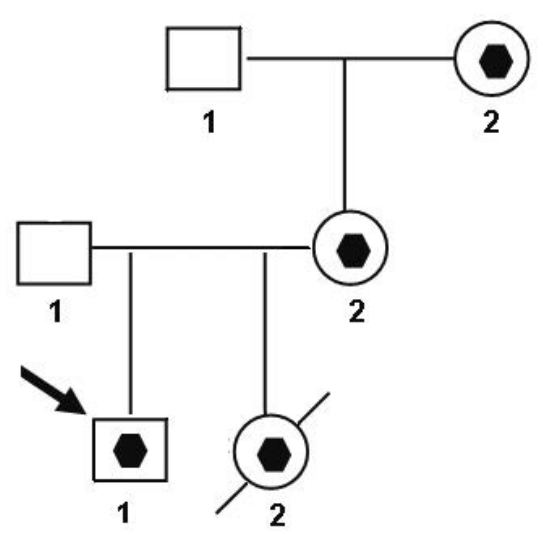

Figure 4. Pedigree of the family. The proband, III-1 (arrow), karyotype was $46, X Y, t(2 ; 4 ; 11 ; 5)(p 21 ; q 34 ; q 21 ; p 15)$ mat. III-2, karyotype was $46, X X$, der $(2 ; 4 ; 5) t(2 ; 4 ; 11 ; 5)(p 21 ; q 34 ; q 21 ; p 15)$ mat. II-2, karyotype was $46, X X, t(2 ; 4 ; 11 ; 5)(\mathrm{p} 21 ; \mathrm{q} 34 ; \mathrm{q} 21 ; \mathrm{p} 15)$ mat. I-2, karyotype was $46, \mathrm{XX}, \mathrm{t}(2 ; 4 ; 11 ; 5)(\mathrm{p} 21 ; \mathrm{q} 34 ; \mathrm{q} 21 ; \mathrm{p} 15$.

typing revealed a karyotype of $46, X X$, der $(2 ; 4 ; 5) t(2 ; 4 ; 11 ; 5)$ (p21;q34;q21;p15)mat (Fig. 5). CMA analysis on uncultured amniocytes detected two pathogenic variants: $\operatorname{arr}[\mathrm{hg} 19]$ 4q34.2q35.2(176,442,864-190,957,460)x1 and $\operatorname{arr}[$ hg19] 11q21q25(92,897,617-134,937,416) x3 (Fig. 6, 7). Ultrasound examination showed cleft lip and palate in the fetus. After genetic counseling, the couple decided to terminate the pregnancy.

\section{DISCUSSION}

About one-third of all CCRs are familial. Familial CCRs tend to have fewer breakpoints and are mainly maternally transmitted via oogenesis, as in the case reported by Binsbergen et al. [1]. Here we present an extremely rare and previously undescribed CCR involving chromosomes 2, 4, 5 and 11. Surprisingly, the CCR was stably transmitted across three generations. The woman inherited this CCR 
III if $k$ !I

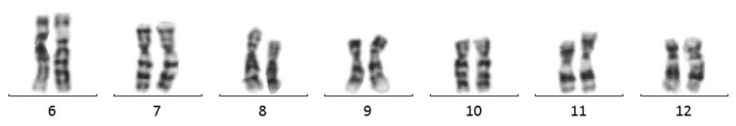

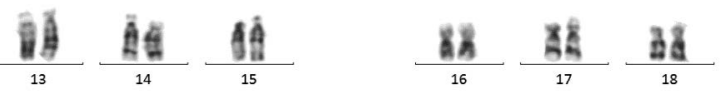

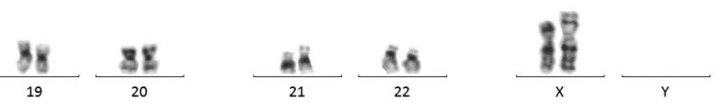

Figure 5. Karyotype of III-2, 46,XX, der(2;4;5)t(2;4;11;5) (p21;q34;q21;p15)mat from her healthy mother and transmitted it to her son, who is phenotypically normal suggesting this is a balanced CCR. However, when the woman was pregnant with her second child, the fetus carries two pathogenic variants and presents with cleft lip and palate (Fig. 6, 7). Our case provides further evidence that balanced CCR carriers can produce abnormal offspring. Therefore, once a CCR is identified, it is necessary to narrow down all the breakpoint regions to determine whether any copy number variants (CNVs) are associated with it. The mechanisms underlying the formation of CCRs remain unknown. There are several events that could lead to complex rearrangements, including replication-based mechanisms and chromothripsis [4]. In our case, the second baby of the CCR carrier has two CNVs, both are close to the breaking region (Fig. 6, 7). Further characterization of these breakpoint junctions in our patient will help understand

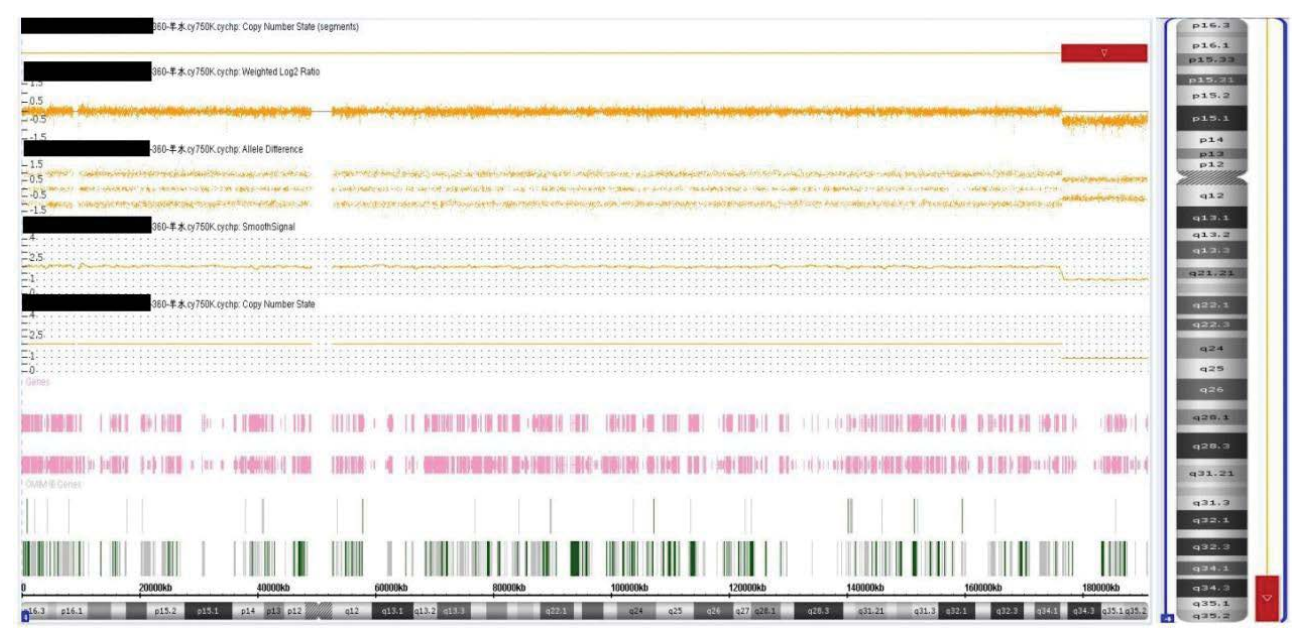

Figure 6. CMA result. 4q34.2q35.2(176,442,864-190,957,460)x1

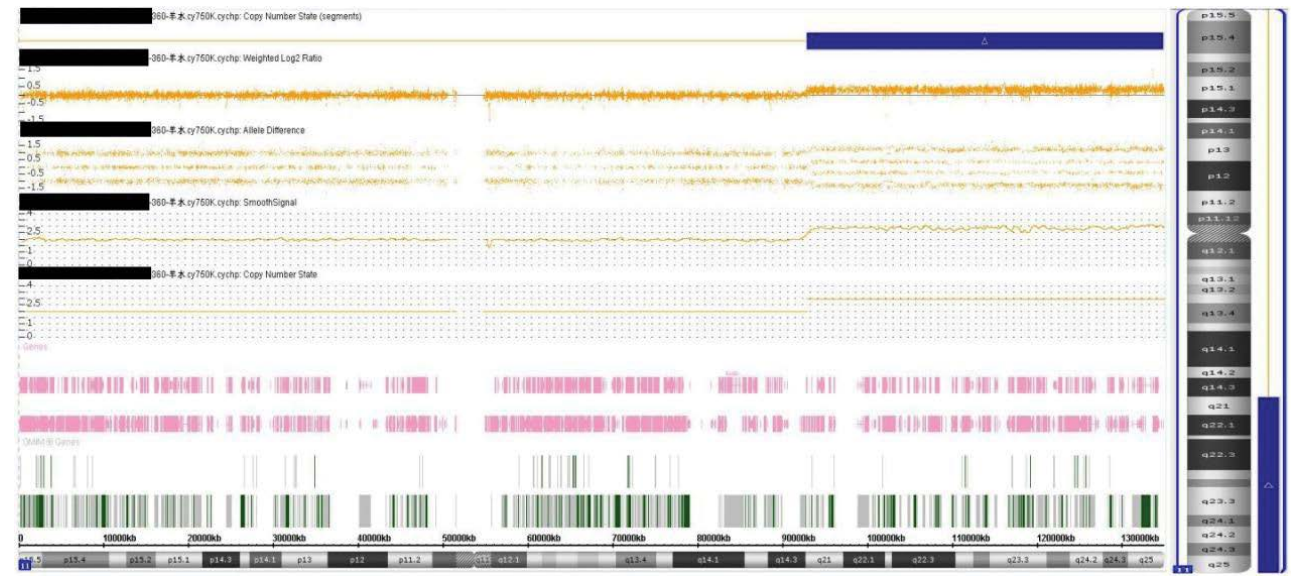

Figure 7. CMA result. 11q21q25(92,897,617-134,937,416)x3 
the molecular mechanisms responsible for the process of this complex CCR.

The outcomes of the CCR carriers are more complex than previously appreciated [6]. Even balanced chromosomal translocations may cause damage or alteration of the functional genes at the breakpoints of the defective chromosomes resulting in the disease phenotype [7]. On the other hand, the possibility of producing normal gametes is theoretically low in CCR carriers. For example, carriers of a balanced chromosomal reciprocal translocation could form a quadriradial chromosome during the pachytene stage of meiosis I. According to the classical gamete formation theory, they can form 18 kinds of gametes and only one is the same balanced chromosomal reciprocal translocation. In our case, the CCR carriers (I-2, II-2 and III-1) could form a'octaradial chromosome' (Fig. 8) during the pachytene stage of meiosis $I$, which will result in $18^{4}=104976$ kinds of gametes. Therefore, there are only one out of 104976 chance that the offspring will carry the same balanced CCR, suggesting there are must be some unknown mechanisms underlying gamete formation.

\section{CONCLUSIONS}

We report an extremely rare and previously undescribed complex chromosomal arrangement that is transmitted

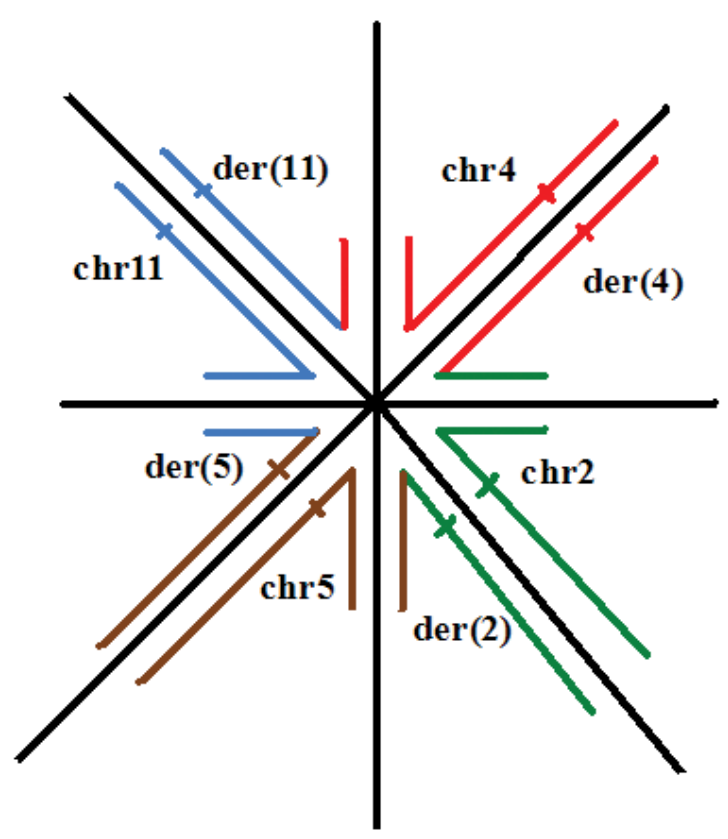

Figure 8. The octaradial chromosome of CCRs (I-2, II-2 and III-1) across three generations. The clinical outcome of this CCR is complex. Careful characterization of all the breakpoint regions is required for prenatal diagnosis and genetic counseling.

\section{Ethics approval and consent to participate}

The research was approved by the Ethics Committee of Renmin Hospital of Wuhan University. All patient guardians gave informed consent to the study.

\section{Acknowledgements}

We are grateful to Dr. John M. Opitz for critically reading the manuscript.

\section{Funding}

This study was supported by the National Natural Science Foundation of China (Grant no. 81773444) and Natural Science Foundation of Hubei Province (Grant no. 2019(FC846).

\section{Consent for publication}

All patient guardians gave informed consent to the publication of this study.

\section{Conflict of interest}

The authors have no conflicts of interest relevant to this article.

\section{REFERENCES}

1. Poot M, Haaf T. Mechanisms of Origin, Phenotypic Effects and Diagnostic Implications of Complex Chromosome Rearrangements. Mol Syndromol. 2015; 6(3): 110-134, doi: 10.1159/000438812, indexed in Pubmed: 26732513.

2. Pellestor F, Anahory T, Lefort $\mathrm{G}$, et al. Complex chromosomal rearrangements: origin and meiotic behavior. Hum Reprod Update. 2011; 17(4): 476-494, doi: 10.1093/humupd/dmr010, indexed in Pubmed: 21486858.

3. Madan K. Balanced complex chromosome rearrangements: reproductive aspects. A review. Am J Med Genet A. 2012; 158A(4): 947-963, doi: 10.1002/ajmg.a.35220, indexed in Pubmed: 22383246.

4. Eisfeldt J, Pettersson M, Vezzi F, et al. Comprehensive structural variation genome map of individuals carrying complex chromosomal rearrangements. PLoS Genet. 2019; 15(2): e1007858, doi: 10.1371/journal. pgen.1007858, indexed in Pubmed: 30735495.

5. Oğlak SC, Bademkıran MH, Obut M. Predictor variables in the success of slow-release dinoprostone used for cervical ripening in intrauterine growth restriction pregnancies. J Gynecol Obstet Hum Reprod. 2020; 49(6): 101739, doi: 10.1016/j.jogoh.2020.101739, indexed in Pubmed: 32251738.

6. Patsalis PC. Complex chromosomal rearrangements. Genet Couns. 2007; 18(1): 57-69, indexed in Pubmed: 17515301.

7. Rao L, Kanakavalli M, Padmalatha V et al. Paternally derived translocation $\mathrm{t}(8 ; 18)(\mathrm{q} 22.1 ; \mathrm{q} 22)$ pat associated in a patient with developmental delay: Case report and review. J Pediatr Neurosci. 2010; 5(1):64-67, doi: 10.4103/1817-1745.66686, indexed in Pubmed: 21042514. 